\title{
PREVALÊNCIA DE INFECÇÃO GENITAL POR CHLAMYDIA TRACHOMATIS NA CONSULTA DE VENEREOLOGIA - ESTUDO DESCRITIVO RELATIVO AO PRIMEIRO SEMESTRE DE 2010
}

\author{
Ana Rita Travassos', João Borges da Costa', Jacinta Azevedo², Irene Santo² \\ 'Clínica Universitária de Dermatologia, Hospital de Santa Maria; ${ }^{2}$ Consulta de DST do Centro de Saúde da Lapa, Lisboa
}

RESUMO - Introdução: A infecção genital por Chlamydia trachomatis ( $\mathrm{C} t$ ) é a infecção sexualmente transmissível bacteriana mais diagnosticada nos Estados Unidos e Reino Unido. É frequentemente assintomática e apresenta uma prevalência mais elevada na população com idade inferior a 25 anos.

Objectivos: Os autores procederam à caracterização epidemiológica, determinação da prevalência da infecção genital por Ct e avaliação dos factores sociodemográficos associados, numa população observada na consulta de Doenças Sexualmente Transmissíveis do Centro de Saúde da Lapa, no primeiro semestre de 2010.

Materiais e Métodos: Todos os indivíduos que acederam à consulta no período de Janeiro-Junho de 2010, testados para $\mathrm{Ct}$ (urina, exsudado uretral/ cervical) foram inseridos no estudo. Os dados foram analisados com nível exacto de significância de $5 \%$.

Resultados: Foram incluídos um total de 968 indivíduos, 642 homens e 326 mulheres. O diagnóstico de C† foi efectuado em 56 doentes, dos quais $75 \%$ eram assintomáticos. As seguintes associações foram encontradas para esta infecção: orientação heterossexual $(p=0,026)$ e, nas mulheres, diagnóstico de condilomas acuminados ou mais de um parceiro sexual nos últimos seis meses. A mediana das idades nas doentes com infecção por Ct foi 25 anos, significativamente inferior à das mulheres sem infecção $(p=0,03)$.

Comentários: Os resultados deste estudo foram semelhantes aos encontrados noutras séries da literatura. Apesar do rastreio anual de Ct ser recomendado pelas guidelines internacionais para IST (CDC/IUSTI) para mulheres com idade igual/ inferior a 25 anos, este ainda não é realizado em Portugal, pelo que a sua implementação deverá ser uma medida a considerar.

PALAVRAS-CHAVE - Chlamydia trachomatis; Infecção sexualmente transmissível; Epidemiologia.

\section{GENITAL CHLAMYDIA TRACHOMATIS INFECTION IN A SEXUALLY TRANSMITTED INFECTION CLINIC - A DESCRIPTIVE STUDY OF THE FIRST SEMESTER OF 2010}

ABSTRACT - Introduction: Chlamydia trachomatis genital infection $(\mathrm{C} t)$ is now the most common sexually transmitted bacterial infection (STI) diagnosed in United Kingdom and in United States and its prevalence is highest in persons younger than 25 years. Asymptomatic infection is also common among both men and women.

Objectives: To assess epidemiological characteristics and prevalence of Ct in a population of patients attending the STI Clinic of Centro de Saúde da Lapa, Lisbon.

Methods: Every patient attending this STI Clinic in the first six months of 2010 and tested to Ct (urine, urethral or vaginal swab) was included in our study. Data was analyzed with an exact significance level of $5 \%$.

Results: A total of 968 patients were included (643 men and 326 women). Ct was diagnosed in 56 patients, of whom $75 \%$ were asymptomatic. The diagnosis of CT was significantly associated to being heterosexual $(p=0,026)$. For women significant association was also found between $\mathrm{Ct}$ diagnosis and the presence of genital warts or having more than one sexual partner during the last six months.

Comments: These results were similar to other European studies. Although the Ct screening is recommended by STI 
guidelines (CDC/ IUSTI), in Portugal it is not routinely performed outside the few existing STI clinics. Knowing the serious consequences of untreated disease, implementation of regular screening in our country should be considered.

KEY-WORDS - Chlamydia trachomatis; Sexually transmitted disease; Epidemiology.

Dr. . Ana Rita Travassos

Hospital de Santa Maria- Centro Hospitalar Lisboa Norte

Avenida Prof. Egas Moniz

1649-028 Lisboa

Tel.: 962341475

Fax: 217954447

E-mail: ritatravassos@gmail.com

\section{INTRODUÇÃO}

O aumento da incidência das Infecções Sexualmente Transmissíveis (IST) acarreta um elevado peso sócio-económico, associado à morbilidade e mortalidade em indivíduos jovens e às suas possíveis implicações ao nível da saúde materno-infantil'.

A infecção genital por Chlamydia trachomatis (Ct) é a IST bacteriana mais frequentemente diagnosticada no Reino Unido e nos Estados Unidos ${ }^{2-4}$, com uma prevalência mais elevada na população com idade inferior a 25 anos $^{2,5}$.

A Ct é uma bactéria intracelular obrigatória que infecta mais de 90 milhões de indivíduos anualmente por transmissão sexual, com uma taxa de transmissão de cerca de $75 \%$,6. É de salientar ainda a transmissão vertical, dada a importância da morbilidade neonatal associada à infecção por Ct.

A Ct têm três biovars (incluindo 15 serovars clássicos e vários serovars adicionais): o trachoma biovar (serovars A-C); urogenital biovar (serovars D-K) e lymphogranuloma venereum (LGV) biovar (serovars L1 -L3) ${ }^{5}$.

Esta infecção é frequentemente assintomática e, na ausência de tratamento, está associada a morbilidade importante em ambos os sexos, nomeadamente infertilidade ${ }^{3,7}$. Na mulher, a infecção é assintomática em cerca de $90 \%$ dos casos, podendo ocasionar: cervicite, uretrite, coitorragias, doença inflamatória pélvica, endometriose, salpingite, gravidez ectópica, parto prematuro, proctite e artrite reactiva ${ }^{3,5}$. Aproximadamente $10 \%$ das mulheres infectadas não tratadas podem desenvolver doença inflamatória pélvica, com elevada taxa de infertilidade subsequente associada ${ }^{5}$.

No homem, a infecção por Ct é assintomática em cerca de $50 \%$ dos casos, podendo estar associada a uretrite, epididimite, proctite e artrite reactiva ${ }^{3,5}$. No recém-nascido a infecção por $\mathrm{Ct}$ pode provocar conjuntivite e pneumonia ${ }^{5}$.

A infecção por C† não é de declaração obrigatória na maioria dos países da União Europeia, incluindo Portugal, pelo que o conhecimento epidemiológico desta infecção nesses países é escasso ${ }^{8,9}$.

Estudos epidemiológicos realizados na Europa confirmam a elevada prevalência de $\mathrm{C} t$ em mulheres jovens com idade inferior a 20 anos, variando de 1,7 a $17 \%$ em mulheres assintomáticas ${ }^{8,9}$. Outros estudos realizados em determinadas populações revelaram as seguintes prevalências: 2-11\% (Alemanha e Austria), 3\% (Basel e Suíça), 2,3\% (Holanda), 5-15\% (União Soviética) e 5,9\% (Hungria), em estudo multicêntrico realizado em mulheres grávidas ${ }^{7} ; 2 \%$ em homens e $3,7 \%$ em mulheres, no contexto de teste anónimo para a infecção HIV em França ${ }^{7}$; $8 \%$ em mulheres que recorreram a consulta para interrupção da gravidez (Islân$\mathrm{dia}^{7} ; 1,4-4,7 \%$ em adolescentes sexualmente activos (16-18 anos de idade) em Antuérpia ${ }^{7}$.

O aumento das taxas de notificação em alguns países da Europa deve ser considerado no contexto da implementação do rastreio da infecção nesses países, assim como da crescente utilização de testes de elevada sensibilidade, como os testes de amplificação de ácido nucleicos (NAATs). 


\section{OBJECTIVOS}

Caracterização epidemiológica da população de uma consulta de IST observada no primeiro semestre de 2010; determinação da prevalência da infecção genital por $\mathrm{Ct}$ e avaliação dos factores associados.

\section{MATERIAIS E MÉTODOS}

Todos os indivíduos que acederam à consulta de Doenças sexualmente transmissíveis (DST) do Centro de Saúde da Lapa no período de Janeiro-Junho de 2010, testados para Ct (urina, exsudado uretral/ cervical) foram inseridos no estudo. A consulta de DST do Centro de Saúde da Lapa é aberta, sem área geográfica definida e com atendimento no próprio dia, sem necessidade de referenciação. A pesquisa de $\mathrm{C}$ té efectuada nos doentes sintomáticos e, por rotina, nos assintomáticos que vêm pela primeira vez à consulta.

Foram consideradas as variáveis sociodemográficas, assim como o diagnóstico de outras IST, sendo analisadas neste trabalho as seguintes: género, idade, orientação sexual, número de parceiros nos últimos seis meses, escolaridade, país de nascimento, outras IST (prévias/actuais) e presença/ausência de sintomas (corrimento uretral/cervical).

Os testes laboratoriais microbiológicos foram realizados no Instituto Nacional de Saúde Dr. Ricardo Jorge, no caso da pesquisa de Ct com recurso ao PCR Cobas-Amplicor (Roche ${ }^{T M}$ ), de acordo com as regras do fabricante. Os doentes com infecção por $\mathrm{Ct}$, bem como os parceiros sexuais por eles referenciados foram tratados com azitromicina $1 \mathrm{~g}$ per os em dose única.

Os dados foram explorados com o package estatístico SPSS 17.0 (SPSS Inc, Chicago, IL; USA). Além da análise exploratória e estatística descritiva dos dados, foram efectuados alguns testes de hipóteses, com uso de nível de significância exacto de $5 \%$. No entanto, por limitações inerentes à amostra, não se pretendeu efectuar extrapolações generalizadas para a população de Lisboa.

$\mathrm{Na}$ análise da independência de duas variáveis qualitativas ou comparação de proporções em grupos independentes, nomeadamente doentes com infecção por Cte indivíduos não infectados, utilizou-se o teste do Qui-quadrado, recorrendo-se ao teste exacto de Fisher quando alguma das frequências esperadas foi inferior a 5 . Na análise das variáveis ordinais ou quantitativas contínuas, como a idade e o número de parceiros entre grupos independentes, optou-se por um teste não paramétrico, que foi o de Mann-Whitney.

\section{RESULTADOS}

Foram incluídos um total de 968 indivíduos 1642 homens; 326 mulheres) e o diagnóstico de Ct foi efectuado em 5,8\% (56/968) destes.

O Quadro I ilustra a caracterização epidemiológica da população da consulta de DST do Centro de Saúde da Lapa durante o período decorrente do estudo (o primeiro semestre de 2010). A mediana das idades foi de 31 anos (32 no sexo masculino e 30 no sexo feminino).

A maioria dos utentes era de origem portuguesa (65\%), 14\% eram oriundos do Brasil e 13\% dos Palop (países africanos de língua oficial portuguesa). A frequência do ensino superior/licenciatura foi referida por $40 \%$ dos utentes, $51 \%$ referiram um único parceiro sexual nos 6 meses anteriores e 37\% (238/642) dos

\section{Quadro I}

\section{CARACTERIZAÇÃO DA POPULAÇÃO DA CONSULTA DE IST ( $1^{\circ}$ SEMESTRE 2010)}

\begin{tabular}{|c|c|c|c|c|}
\hline & Masc. & Fem. & Total \\
\hline & & 642 & 326 & 968 \\
\hline \multicolumn{2}{|c|}{ Idade (mediana) } & 32 & 30 & 31 \\
\hline \multirow{5}{*}{$\begin{array}{l}\text { País de } \\
\text { Origem }\end{array}$} & Portugal & 418 & 212 & 630 \\
\hline & Europa & 43 & 19 & 62 \\
\hline & PALOP & 80 & 45 & 125 \\
\hline & Brasil & 88 & 48 & 136 \\
\hline & Outros & 13 & 2 & 15 \\
\hline \multirow{4}{*}{ Escolaridade } & Primária & 175 & 89 & 264 \\
\hline & Secundário & 214 & 102 & 316 \\
\hline & Superior & 251 & 135 & 386 \\
\hline & Desconhecido & 2 & 0 & 2 \\
\hline \multirow{2}{*}{$\begin{array}{l}\text { Orientação } \\
\text { sexual }\end{array}$} & Homo/Bissexual & 238 & 7 & 245 \\
\hline & Heterossexual & 404 & 319 & 723 \\
\hline \multirow{4}{*}{$\begin{array}{l}N^{\circ} \text { parceiros } \\
(6 \mathrm{~m})\end{array}$} & 0 & 7 & 25 & 32 \\
\hline & 1 & 281 & 211 & 492 \\
\hline & 2 a 4 & 270 & 70 & 340 \\
\hline & $>=5$ & 84 & 20 & 104 \\
\hline \multicolumn{2}{|c|}{ Prática prostiłuição } & 1 & 20 & 21 \\
\hline \multicolumn{2}{|c|}{ Consumo drogas e.v. } & 8 & 3 & 11 \\
\hline
\end{tabular}




\section{GEIDST}

homens referia ter sexo com homens. Um homem e 20 mulheres praticavam prostituição e oito homens e três mulheres eram utilizadores de drogas endovenosas.

O Quadro II caracteriza os 56 doentes com diagnóstico de infecção genital por Clamídia $(\mathrm{Ct+})$, dos quais $57 \%(32 / 56)$ são homens e $43 \%(24 / 56)$ mulheres. Dos doentes $\mathrm{C} t+75 \%$ eram assintomáticos $(81 \%$ dos homens e $67 \%$ das mulheres).

\section{Quadro II}

\begin{tabular}{|c|c|c|c|c|}
\hline \multicolumn{5}{|c|}{ CARACTERIZAÇÃO DOS INDIVÍDUOS CT+ } \\
\hline & & Masc. & Fem. & Total \\
\hline & & 32 & 24 & \\
\hline \multicolumn{2}{|c|}{$\begin{array}{l}\text { Sintomáticos } \\
\text { (corrimento uretral/vaginal) }\end{array}$} & 6 & 8 & 14 \\
\hline \multicolumn{2}{|c|}{ Idade (mediana) } & 32 & 25 & 26 \\
\hline \multirow{5}{*}{$\begin{array}{l}\text { País de } \\
\text { Origem }\end{array}$} & Portugal & 16 & 15 & 31 \\
\hline & Europa & 2 & 3 & 5 \\
\hline & PALOP & 10 & 3 & 13 \\
\hline & Brasil & 2 & 2 & 4 \\
\hline & Outros & 2 & 1 & 3 \\
\hline \multirow{3}{*}{ Escolaridade } & Primária & 8 & 7 & 15 \\
\hline & Secundário & 15 & 10 & 25 \\
\hline & Superior & 9 & 7 & 16 \\
\hline \multirow{2}{*}{$\begin{array}{l}\text { Orientação } \\
\text { sexual }\end{array}$} & Homo/Bissexual & 7 & 0 & 7 \\
\hline & Heterossexual & 25 & 24 & 49 \\
\hline \multirow{4}{*}{$\begin{array}{l}N^{\circ} \text { parceiros } \\
(6 \mathrm{~m})\end{array}$} & 0 & 0 & 0 & 0 \\
\hline & 1 & 14 & 13 & 27 \\
\hline & 2 a 4 & 14 & 9 & 23 \\
\hline & $>=5$ & 4 & 2 & 6 \\
\hline
\end{tabular}

A mediana das idades nos doentes com diagnóstico de Ct foi de 26 anos: 32 anos nos homens e 25 anos nas mulheres, esta última significativamente inferior à mediana das idades das mulheres Ct negativas (Mann-Whitney $=5,151 ; p=0,03$ ).

A maioria dos doentes com infecção era de origem portuguesa (55\%) e $23 \%$ oriundos dos Palop; $45 \%$ tinha frequência do ensino secundário e $28 \%$ frequência universitária/ licenciatura.
A totalidade das mulheres com diagnóstico de $\mathrm{C} t$ e $78 \%$ dos homens infectados eram heterossexuais, tendo sido encontrada uma relação estatisticamente significativa entre a infecção por $\mathrm{Ct}$ e a orientação heterossexual $(p=0,026)$.

Quanto ao número de parceiros sexuais, $48 \%$ $(27 / 56)$ referiram um parceiro sexual e $52 \%(29 / 56)$ mais de um parceiro nos últimos seis meses, tendo sido encontrada uma relação estatisticamente significativa com o número de parceiros sexuais superior a um no grupo das mulheres $(p=0,038)$.

O Quadro III mostra as IST diagnosticadas previamente ou concomitantes com a infecção por $\mathrm{Ct}$ (co-infecções): $11 \%$ tinham um diagnóstico anterior de IST (11 homens e uma mulher) e 38\% (12 homens e 9 mulheres) tinham uma co-infecção. Nas mulheres com infecção por $\mathrm{Ct}$, foi encontrada uma relação estatisticamente significativa com a presença de condilomas anogenitais $(p=0,015)$.

\section{Quadro III}

\section{ANTECEDENTES/ CO-INFECÇÃO DE IST EM DOENTES CT+}

\begin{tabular}{|c|c|c|c|c|}
\hline & Masc. & Fem. & Total \\
\hline & & 32 & 24 & \\
\hline \multirow{7}{*}{$\begin{array}{l}\text { Antecedentes } \\
\text { de IST }\end{array}$} & & 5 & 1 & 6 \\
\hline & $\mathrm{Ct}$ & 2 & 0 & 2 \\
\hline & Gonorreia & 1 & 0 & 1 \\
\hline & Sífilis & 1 & 0 & 1 \\
\hline & $\begin{array}{l}\text { Condilomas } \\
\text { anogenitais }\end{array}$ & 0 & 1 & 1 \\
\hline & Herpes genital & 2 & 0 & 2 \\
\hline & Infecção HIV & 4 & 0 & 4 \\
\hline \multirow{8}{*}{$\begin{array}{l}\text { Co-infecção } \\
\text { (outras IST) }\end{array}$} & & 12 & 9 & 21 \\
\hline & Gonorreia & 2 & 1 & 3 \\
\hline & Sífilis recente & 4 & 0 & 4 \\
\hline & $\begin{array}{l}\text { Sífilis de duração } \\
\text { indeterminada }\end{array}$ & 1 & 0 & 1 \\
\hline & $\begin{array}{l}\text { Condilomas } \\
\text { anogenitais }\end{array}$ & 3 & 7 & 10 \\
\hline & Herpes genital & 3 & 1 & 4 \\
\hline & Tricomonose & 0 & 2 & 2 \\
\hline & Infecção HIV & 1 & 0 & 1 \\
\hline
\end{tabular}




\section{COMENTÁRIOS}

Os resultados deste estudo foram semelhantes aos encontrados noutras séries da literatura ${ }^{2,3,7-10}$.

O presente estudo não é representativo da população portuguesa, nem mesmo da população da cidade de Lisboa, tal como mencionado noutro estudo semelhante ${ }^{10}$, dadas as características dos utentes da Consulta: população jovem, elevado nível educacional e grande percentagem de homens que referem sexo com homens. A relevância deste estudo prende-se com o facto de nesta consulta, graças à sua metodologia e ao rastreio sistemático dos utentes, ser possível estudar e comparar indivíduos $\mathrm{C}++$ (sintomáticos e assintomáticos) e restante população Ct negativa.

Os nossos dados indicam uma prevalência elevada de infecção por $\mathrm{Ct}(5,8 \%)$, particularmente em mulheres jovens, com uma mediana das idades nas doentes $\mathrm{Ct}+$ (25 anos) inferior às outras mulheres Ct- (Mann-Whitney $=5,151 ; p=0,03)$. Este padrão etário é semelhante ao referido noutros estudos realizados em Portuga $\left.\right|^{8,10} \mathrm{e}$ noutros países $2,3,7,9$.

A infecção por Ct foi também associada à orientação heterossexual $(p=0,026)$ e ao número de parceiros sexuais superior a um $(p=0,038)$, como também é referido na literatura ${ }^{3}$.

A associação de infecção por Ct à presença de condilomas anogenitais nas mulheres $(p=0,015)$, também já foi referida num estudo realizado numa população semelhante, da mesma consulta de DST do Centro de Saúde da Lapa ${ }^{10}$.

O elevado número de infecções assintomáticas na nossa série $(75 \%)$ está de acordo com o referido na literatura ${ }^{2,3,7}$ e sublinha a necessidade de realizar uma procura de casos ou rastreio em determinadas populações.

Apesar do rastreio anual de $\mathrm{Ct}$ ser recomendado pelas orientações internacionais para IST (CDC) IUSTI) $)^{4,5}$ para mulheres com idade igual ou inferior a 25 anos e na gravidez ( $1 \circ$ e $3^{\circ}$ trimestre), este não é realizado em Portugal, pelo que a sua implementação é necessária.

GEIDST

\section{BIBLIOGRAFIA}

1. Santo I, Azevedo J, Cardoso J: A consulta de doenças sexualmente transmissíveis do centro de saúde da Lapa. caracterização da população utente e padrão epidemiológico das IST. Trab Soc Port Dermatol Venereol 65(3): 383-94 (2007).

2. Adams EJ; Charlett A, Edmunds WJ, Hughes G: Chlamydia trachomatis in the United Kingdom: a systematic review and analysis of prevalence studies. Sex Trasm Infect 80: 354-62 (2004).

3. LaMontagne DS, Fenton KA, Randall S, Anderson S, Carter P: Establishing the national Chlamydia screening programme in England: results from the first year of screening. Sex Transm Infect 80: 335-41 (2004).

4. Centers for Disease Control and Prevention: Sexually Transmitted Diseases Treatment Guidelines, 2010. MMWR 59, pp 44-48, 2010.

5. Lanjouw Ossewaarde JM, Stary A, Boag F:European guideline for the management of Chlamydia trachomatis infections. Van der Meijden, WI(ed). disponível em: www.iusti.com (2010).

6. Markos AR: The concordance of Chlamydia trachomatis genital infection between sexual partners, in the era of nucleic acid testing. Sex Health 2: 23-4 (2005).

7. Adler MV, Meheust AZ: Epidemiology of sexually transmitted infections and human immunodeficiency virus in Europe. J Eur Acad Dermatol Venereol 14: 370-7 (2000).

8. Azevedo J, Santo I, Gomes JP, Viegas S, Florindo C, Nunes B, Borrego MJ: Co-infecção genital por Neisseria gonorrhoeae e Chlamydia trachomatis em utentes de uma consulta de doenças sexualmente transmissíveis. Trab Soc Port Dermatol Venereol 64(4): 495-500 (2006).

9. Fenton KA, Lowndes CM: Recent trends in the epidemiology of sexually transmitted infections in Europe Union. Sex Transm Infect 80: 255-63 (2004).

10. Borges da Costa J, Azevedo J: Sexually transmitted infections and related sociodemographic factors in Lisbon's major Venereology Clinic: a descriptive study of the first 4 months of 2007. J Eur Acad Dermatol Venereol 24(7): 811-4 (2010). 